\title{
High detection rate of osteoporosis with screening of a general hospitalized population: a 6-year study in 6406 patients in a university hospital setting
}

Olivier Malaise ${ }^{1 *}$ (D) Marie Detroz ${ }^{1}$ (D) Mathieu Leroy ${ }^{1}$ (D), Lorenzo Leonori ${ }^{1}$ (D) Laurence Seide $^{2}$ (D) and Michel G. Malaise ${ }^{1}$ (D)

\begin{abstract}
Background: Osteoporosis is a highly prevalent disease identified by Dual Energy X-ray Absorptiometry (DEXA) that can be performed in an ambulatory (out-patient) or hospitalized population. We evaluated the use of baseline inhospital DEXA screening to identify osteoporosis in ambulatory care and hospitalized patients; we also assessed specific risk factors for osteoporosis among these populations.

Methods: We included a baseline initial DEXA from 6406 consecutive patients at our tertiary referral University Hospital.

Results: Osteoporosis was diagnosed in $22.3 \%$ of the study population. In univariate analysis, osteoporosis risk factors were age, fracture history and low BMI (for all 3 sites), but also corticotherapy (lumbar spine and femoral neck) and male (lumbar spine). In multivariate analysis, age, fracture history, low BMI, and male increased osteoporosis risk. In-hospital screening yielded a higher percentage of osteoporosis positive scans than ambulatory care screening (31.8\% vs $18.5 \%, p<0.001)$. In-hospital screening targeted an older and more predominantly male population with a higher fracture history. Z-scores revealed that this difference was not only due to an older age of the population and mainly concerned cortical bone.

Conclusions: In-hospital osteoporosis screening revealed more osteoporosis than screening in ambulatory practice and could be an additional tool to improve the identification and management of osteoporosis. In addition to typical risk factors, we identified male gender as associated with osteoporosis detection in our cohort.
\end{abstract}

Keywords: Osteoporosis, Dual energy X-ray absorptiometry, Hospitalization, Male

\section{Background}

Osteoporosis is a disease characterized by low bone mass and deterioration of bone micro-architecture, leading to fragility and an increased fracture risk. Osteoporosis has a high prevalence in women in industrialized countries, ranging from 9 to $15 \%$ based on total hip bone mineral density (BMD) and $16-38 \%$ when vertebral BMD was included [1]. Osteoporosis also affects males, albeit at a decreased rate, with a global prevalence of $3 \%$

\footnotetext{
* Correspondence: olivier.malaise@chuliege.be

1 Departments of Rheumatology, University Hospital of Liège, Liège University, Liège, Belgium

Full list of author information is available at the end of the article
}

(hip) and 8\% (vertebra) [1]. Between 2005 and 6 and 2013-14, there was a decline in bone mass density in older US adults, indicating that osteoporosis prevalence is not decreasing and is far from being resolved [2]. BMD is strongly correlated to fracture risk [3]. The risk of vertebral or femoral neck fracture was 18.0 and 28.0 times higher, respectively, when women after 50 years old were osteoporotic [4]. Ten to $20 \%$ of patients with hip fracture died in the first year following the fracture with a risk of premature mortality that remains elevated for at least 10 years $[5,6]$.

Osteoporosis is preventable and treatable using the various pharmacological therapies available and this 
management reduces fracture rates [7, 8]. Dual-Energy $\mathrm{X}$-ray Absorptiometry (DEXA) scan is the cornerstone of osteoporosis screening, while the Fracture Risk Assessment Tool (FRAX) score adds valuable further information when evaluating fracture risk $[9,10]$. Despite the clear links between osteoporosis and fracture, screening rates are low in primary care with only 21.1, 26.5 and $12.8 \%$ of women aged 50-64, 65-79 and $>80$ years, respectively, being screened in a large US study [5], while only $10.8 \%$ of women $>65$ years underwent osteoporosis screening in a US primary care setting [11]. Even after hip fracture, the screening rate remains low with only 17.1 and $23.1 \%$ of women undergoing osteoporosis assessment/treatment within 6 and 12 months of their fracture, respectively [12]. Data from other countries also confirm the lack of osteoporosis screening [13]. As a consequence of this lack of primary and secondary prevention, emphasis has recently been placed on improved screening efforts after an initial fracture event, such as the development of "fracture liaison services", that are effective in reducing second fractures [14]. To improve osteoporosis screening, opportunistic use of CT scan has also been proposed to analyze BMD [15] and detect osteoporotic fractures [16], as well as FRAX algorithm utilization [17].

As a population, hospitalized patients have variable comorbid conditions and receive pharmacotherapies that can have deleterious effects on bone thereby leading to osteoporosis. Targeting such hospitalized patients could represent an opportunity to improve the detection of osteoporosis. Hence, we studied the effectiveness of inhospital osteoporosis screening using DEXA ( $\mathrm{T}$ and Zscores: lumbar spine; femoral neck, total hip) and risk factor scores, and compared the osteoporosis rate in a hospitalized sub-population with that of patients screened in the ambulatory care setting.

\section{Methods \\ Patients}

The Department of Rheumatology of the University Hospital of Liège (Belgium) offers DEXA studies to ambulatory outpatients prescribed by general practitioners or by medical specialists during clinic-based consultation or to hospitalized in-patients in various departments (e.g. rheumatology, neurology, internal medicine, endocrinology ...).

\section{DEXA procedure}

All the examinations were performed by the same Discovery A DEXA system (Hologic ${ }^{\circ}$, Bedford, MA, USA), with lumbar spine (L1-L4), total hip and femoral neck analysis. For total hip and femoral neck, the left side was analyzed except when prosthetic material was present. T-scores and Z-scores were reported for these three sites. T-score values were considered as normal if $>-1$, osteopenic if $\leq$
-1 and $>-2.5$ and osteoporotic if $\leq-2.5$. Z-score were also categorized in different ranks: $<-1,<-2$ and $\leq-2.5$. The proportion of patients with normal values, osteopenia or osteoporosis was analyzed for each year and for the global period (2007-2012). The same specialized nurse (ML) or physiotherapist (LL) is in charge of the DEXA determination, whatever the origin of the patients. Standardization procedures were performed according to the International Society for Clinical Densitometry. In particular, during the years analyzed, daily quality control with phantom were performed to ensure that these values were located at maximum $+/-1.5 \%$ of the mean value of calibration. About the parameters of the DEXA (Hologic ${ }^{\circ}$, Bedford, MA, USA), the total bone mineral density (BMD) coefficient of variation $(\mathrm{CV})=1.0 \%$.

\section{Study protocol}

We conducted a retrospective study to analyse our DEXA results in real-life conditions. Approval was obtained from the local ethical committee of the University Hospital of Liège (reference 2019/152). We only considered DEXA scan that were realized our Discovery A DEXA system in our instution, and not DEXA scans that could be realized in another center. Data were available for the 2007-2012 period and 9354 DEXA scan were performed in our hospital during that period. The only specific exclusion criteria was age $<18$ years. During the period analyzed, only the patient's initial DEXA study was assessed and subsequent DEXA scans were not included. After application of these criteria, 6406 first DEXA examinations were included in the study database. Data concerning sex, age, body mass index (BMI) and history of previous fracture (whatever the site) were collected. Medication data included history of glucocorticoid (GC) use as defined by FRAX (current or previous treatment for $>3$ months at a prednisone dose $\geq 5$ $\mathrm{mg} /$ day) and proton-pump inhibitors (PPI) use. All data was de-identified in the study database: data will be made anonymous as soon as it is encoded and stored in a locked cabinet. Only the investigators of the study will have access to the data collected.

To assess whether any differences existed in terms of the diagnosis rates for osteoporosis, we divided our study population ( $n=6406$ patients) in two groups: ambulatory care and hospitalized in-patients.

\section{Statistical analysis}

Results were presented as mean \pm standard deviation (SD) or as median and range (minimum-maximum) for continuous variables and as frequency tables for qualitative variables. Comparisons between ambulatory and hospitalized patients were performed using Student's ttest for continuous variables and the chi-square test for the categorical variables. Univariate logistic regression 
models investigated the relationship between osteoporosis risk and demographic variables (age, BMI, sex GC use, PPI intake and previous fracture). Multivariate logistic regression model was applied on these factors and the year of the DEXA scan. The results were considered significant at the uncertainty level of $5 \% \quad(p<0.05)$. Calculations were performed using SAS software version 9.4 (SAS Institute, Cary, NC, USA).

\section{Results}

\section{Demographics}

Six thousand four hundred six first DEXA examinations were included (2007: $n=1494 ; 2008: n=1158 ; 2009: n=$ 1079; 2010: $n=945 ; 2011: n=868 ; 2012: n=862)$. In the 6406 patients that had a first DEXA scan, the mean \pm SD $(\min , \max )$ age was $60.5 \pm 14.3(18,98)$ years and the mean \pm SD (min, max) BMI was $25.2 \pm 5.1(11.7,60.4)$ $\mathrm{kg} / \mathrm{m}^{2}$. In total, $74.4 \%$ of patients were female. Overall, $30.5 \%$ of the patients had a history of GC use and $28.7 \%$ were taking a PPI. A previous fracture was recorded in $29.0 \%$ of patients. At the time of DEXA examination, 4561 patients (71.2\%) were ambulatory and 1845 (28.8\%) patients were hospital in-patients.

\section{DEXA results}

Normal, osteopenia and osteoporosis rates in the overall study population are shown in Table 1 . The median (min, max) T-scores were $-1.0(-6.0,8.0)$ for lumbar spine, $-1.3(-5.2,3.8)$ for femoral neck and $-1.0(-5.2$, 6.0) for total hip, respectively. Osteoporosis at least one of the three sites was identified in 1429/6406 patients (22.3\%). At each anatomical location osteoporosis rates were as follows: $13.7 \%$ at the lumbar spine; $13.6 \%$ at the femoral neck and $9.2 \%$ at the total hip (Table 1). Lastly, 252 of 6406 patients (3.9\%) had osteoporosis at all the three sites (Table 1).

Overall, patients with normal T-scores, osteopenia or osteoporosis on DEXA had a statistically different mean age (SD) of 56.4 (13.9), 60.6 (13.8) and 65.3 (14.4) years old, respectively ( $p<0.001$ for each comparison). Figure 1 shows the percentage of normal, osteopenic and osteoporotic patients according to the year of DEXA examination for the whole population. The percentage of osteoporotic patients changed according to a quadratic model with time (linear term $p=0.0025$, quadratic term: $p=0.022$ ) with variations from year to year; the percentage of osteopenic patients remained stable $(p=0.95)$ throughout the study period (Fig. 1).

\section{Osteoporosis risk factors}

In univariate analysis for the whole population $(n=$ 6406), risks factor for osteoporosis (independently of the anatomical site, i.e. T-score $\leq-2.5$ at the lumbar spine, total hip or femoral neck) were older age, a lower BMI,
Table 1 Osteoporosis diagnosis in the whole population (20072012)

\begin{tabular}{|c|c|c|c|}
\hline & Categories & $N$ & Number (Percent) \\
\hline \multirow[t]{4}{*}{ Global diagnostic } & & 6406 & \\
\hline & Normal & & $1724(26.9)$ \\
\hline & Osteopenia & & $3253(50.8)$ \\
\hline & Osteoporosis & & $1429(\mathbf{2 2 . 3})$ \\
\hline \multirow[t]{4}{*}{ Lumbar spine diagnostic } & & 6377 & \\
\hline & Normal & & $3121(48.9)$ \\
\hline & Osteopenia & & $2380(37.3)$ \\
\hline & Osteoporosis & & $876(13.7)$ \\
\hline \multirow[t]{4}{*}{ Femoral neck diagnostic } & & 6262 & \\
\hline & Normal & & $2277(36.4)$ \\
\hline & Osteopenia & & $3134(50.0)$ \\
\hline & Osteoporosis & & $851(\mathbf{1 3 . 6 )}$ \\
\hline \multirow[t]{4}{*}{ Total hip diagnostic } & & 6278 & \\
\hline & Normal & & $3033(48.3)$ \\
\hline & Osteopenia & & $2668(42.5)$ \\
\hline & Osteoporosis & & $577(\mathbf{9 . 2})$ \\
\hline \multirow[t]{3}{*}{ Osteoporosis at the three sites } & & 6406 & \\
\hline & No & & $6154(96.1)$ \\
\hline & Yes & & $252(3.9)$ \\
\hline
\end{tabular}

GC intake and a previous fracture (Table 2). The risk factors were also described separately for each anatomical site: older age, lower BMI and a previous fracture were associated with osteoporosis at all three anatomical sites, while GC intake was only correlated with lumbar spine and femoral neck. Female was associated with osteoporosis detection at the total hip, and male for the lumbar spine (Table 2).

A multivariate analysis for the global osteoporosis risk included seven variables: year of the DEXA scan, age, BMI, sex, GC use, PPI intake and previous fracture. This analysis included 4090 of the initial population with complete data for all variables. In this multivariate

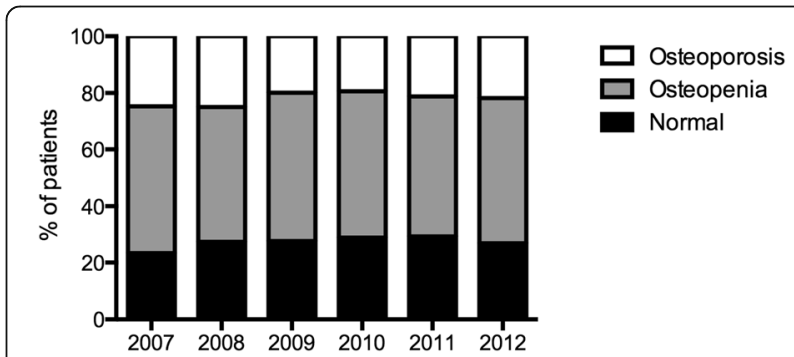

Fig. 1 Percentage of normal, osteopenic and osteoporotic patients according to the year of DEXA examination for the whole population 
Table 2 Risk factors for osteoporosis in univariate (left) and multivariate (right) analysis in the whole population (2007-2012)

\begin{tabular}{|c|c|c|c|c|c|c|}
\hline & OR & IC95\% & $p$-value & OR & IC95\% & $p$-value \\
\hline \multicolumn{7}{|c|}{ Global analysis } \\
\hline Age (years) & 1.03 & $1.03-1.04$ & $<0.0001$ & 1.03 & $1.02-1.03$ & $<0.0001$ \\
\hline BMI $\left(\mathrm{kg} / \mathrm{m}^{2}\right)$ & 0.88 & $0.87-0.90$ & $<0.0001$ & 0.86 & $0.84-0.87$ & $<0.0001$ \\
\hline Sex (male) & 1.12 & $0.98-1.28$ & 0.094 & 1.23 & $1.02-1.47$ & 0.029 \\
\hline GC use & 1.20 & $1.06-1.36$ & 0.0051 & 1.18 & $0.98-1.41$ & 0.084 \\
\hline PPI intake & 0.99 & $0.85-1.17$ & 0.95 & 0.97 & $0.81-1.17$ & 0.77 \\
\hline Previous fracture & 3.20 & $2.83-3.62$ & $<0.0001$ & 2.80 & $2.36-3.32$ & $<0.0001$ \\
\hline \multicolumn{7}{|c|}{ Lumbar spine } \\
\hline Age (years) & 1.02 & $1.01-1.02$ & $<0.0001$ & 1.01 & $1.00-1.02$ & 0.010 \\
\hline BMI $\left(\mathrm{kg} / \mathrm{m}^{2}\right)$ & 0.91 & $0.90-0.93$ & $<0.0001$ & 0.90 & $0.88-0.92$ & $<0.0001$ \\
\hline Sex (male) & 1.52 & $1.30-1.77$ & $<0.0001$ & 1.78 & $1.45-2.18$ & $<0.0001$ \\
\hline GC use & 1.18 & $1.01-1.37$ & 0.032 & 1.06 & $0.86-1.31$ & 0.60 \\
\hline PPI intake & 0.91 & $0.74-1.11$ & 0.35 & 0.90 & $0.72-1.11$ & 0.33 \\
\hline Previous fracture & 2.56 & $2.22-2.97$ & $<0.0001$ & 2.47 & $2.03-3.02$ & $<0.0001$ \\
\hline \multicolumn{7}{|c|}{ Femoral neck } \\
\hline Age (years) & 1.05 & $1.04-1.06$ & $<0.0001$ & 1.05 & $1.04-1.06$ & $<0.0001$ \\
\hline BMI $\left(\mathrm{kg} / \mathrm{m}^{2}\right)$ & 0.84 & $0.83-0.86$ & $<0.0001$ & 0.79 & $0.77-0.82$ & $<0.0001$ \\
\hline Sex (male) & 0.87 & $0.74-1.04$ & 0.12 & 0.90 & $0.71-1.15$ & 0.39 \\
\hline GC use & 1.20 & $1.03-1.40$ & 0.019 & 1.31 & $1.03-1.65$ & 0.025 \\
\hline PPI intake & 1.08 & $0.89-1.32$ & 0.43 & 1.09 & $0.87-1.37$ & 0.47 \\
\hline Previous fracture & 3.63 & $3.13-4.21$ & $<0.0001$ & 2.95 & $2.38-3.66$ & $<0.0001$ \\
\hline \multicolumn{7}{|c|}{ Total hip } \\
\hline Age (years) & 1.05 & $1.04-1.06$ & $<0.0001$ & 1.04 & $1.03-1.05$ & $<0.0001$ \\
\hline BMI $\left(\mathrm{kg} / \mathrm{m}^{2}\right)$ & 0.82 & $0.80-0.84$ & $<0.0001$ & 0.78 & $0.75-0.80$ & $<0.0001$ \\
\hline Sexe (male) & 0.68 & $0.55-0.85$ & 0.0004 & 0.87 & $0.65-1.16$ & 0.33 \\
\hline GC use & 1.13 & $0.94-1.36$ & 0.20 & 1.19 & $0.89-1.57$ & 0.24 \\
\hline PPI intake & 0.90 & $0.70-1.15$ & 0.38 & 0.87 & $0.65-1.15$ & 0.32 \\
\hline Previous fracture & 4.58 & $3.83-5.48$ & $<0.0001$ & 3.79 & $2.92-4.92$ & $<0.0001$ \\
\hline
\end{tabular}

analysis we observed significant effects of age [odds ratio (OR), (95\% confidence interval): 1.03 (1.02-1.03), $p<$ 0.0001], BMI [OR: 0.86 (0.84-0.87), $p<0.0001$ ], male [1.23 (1.02-1.47), $p=0.029]$ and an history of a previous fracture [2.80 (2.36-3.32), $p<0.0001]$. Age, BMI and fracture were significant risk factors for each anatomical site separately [OR (IC95\%) at lumbar spine: 1.01 (1.001.02), 0.90 (0.88-0.92), 2.47 (2.03-3.02) respectively; OR at femoral neck: 1.05 (1.04-1.06), 0.79 (0.77-0.82), 2.95 (2.38-3.66) respectively; OR total hip1.04 (1.03-1.05), $0.78(0.75-0.80), \quad 3.79(2.92-4.92)$ respectively $(p<$ 0.0001 for all expect for age and lumbar spine with $p=$ $0.01)]$. GC use was significant associated with OP at the femoral neck [OR $1.31(1.03-1.65), p=0.025$ ] and male with OP at lumbar spine [OR 1.78 (1.45-2.18), $p<$ $0.0001]$. These data were described in Table 2.

\section{Comparisons between ambulatory and hospitalized patients}

On the 6406 patients, 4561 (71.2\%) were ambulatory and 1845 (28.8\%) were hospitalized. In-patients originated from rheumatology (21.8\%), neurology (15.5\%), internal medicine $(14.7 \%)$, endocrinology $(11.8 \%)$, orthopedic surgery (5.7\%), nephrology (5.5\%), pulmonary (4.4\%), cardiology (4.0\%), neurosurgery (3.8\%) and abdominal surgery $(3.4 \%)$ representing $90.6 \%$ of the cohort $(77.7 \%$ from medical departments and $12.9 \%$ from surgical departments). Percentage of osteoporotic patients by 
department in the hospitalized sub-population was represented in Additional file 1: Figure S1, with the highest percentage for the pneumology department. T-score [median (inter-quartile range)] at the lumbar spine, the femoral neck and the total hip were significantly lower among the hospitalized patients. At each anatomical site the T-scores were as follows: lumbar spine: $-1.0(-1.9$; $0.1)$ vs. $-1.1(-2.1 ; 0.0)(p=0.0001)$; femoral neck -1.2 $(-1.9 ;-0.5)$ vs. $-1.5(-2.3 ;-0.7)(p<0.0001)$ and total hip $-0.9(-1.6 ;-0.2)$ vs. $-1.3(-2.1 ;-0.5)(p<0.0001)$ in the ambulatory and in-patient groups, respectively.

For the diagnosis of osteoporosis, this was present at at least one site in $18.5 \%$ of the ambulatory patients, but this rose significantly to $31.8 \%$ in the hospitalized population $(<0.0001)$ (Table 3). Lumbar spine, femoral neck and total hip were also separately analyzed, with significantly more osteoporosis diagnosed for each site in the in-patient group (lumbar spine (\%): 11.4 vs 19.1; femoral neck 10.4 vs 21.5 ; total hip 6.2 vs 16.6 for ambulatory and hospitalized patients respectively, all $p<0.0001$ ). A total of $2.3 \%$ of the ambulatory patients had osteoporosis at all the three sites, while this percentage was nearly 3.5 times higher $(7.9 \%)(p<0.0001)$ in hospitalized patients. The differences in osteoporosis diagnosis between ambulatory and hospitalized patients over each year remained stable throughout the analysis period (Fig. 2).

Demographic data and risk factors for osteoporosis were also different between ambulatory and hospitalized patients (Table 4): hospitalized patients were significantly older $(p<0.0001)$ and more had a previous fracture $(p<0.0001)$. Males were significantly higher in proportion in hospitalized patients $(p<0.0001)$. BMI was

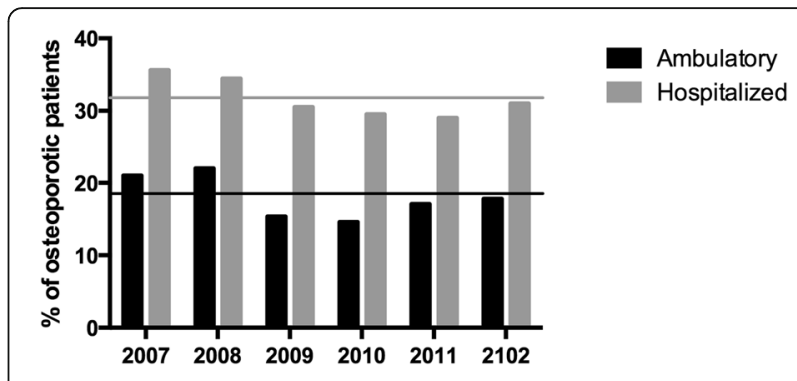

Fig. 2 Comparison between the percentage of osteoporotic patients by year in ambulatory and hospitalized patients. The grey horizontal line represented the 2007-2012 mean percentage in the hospitalized sub-population. The black horizontal line represented the percentage in the ambulatory sub-population

also higher in hospitalized patients $(p=0.028)$. There was no difference in terms of history of GC use.

To determine whether the difference between T-score was related only to greater age in the hospitalized versus the ambulatory patient group (mean ages 64.2 vs 59.0 years, respectively), Z-scores were also compared. Zscores at the femoral neck and the total hip were significantly lower $(p<0.0001)$ in hospitalized vs ambulatory patients; $\mathrm{Z}$ scores at the lumbar spine were lower in the hospitalized group, but the difference was not significant $(p=0.076)$. When Z-scores were ranked as $<-1,<-2,<$ -2.5 , the hospitalized group had significantly lower ranked scores than the ambulatory group $(p<0.0001)$ irrespective of the anatomical site studied (Additional file 2: Table S1). Z-score ranks of $<-1,<-2$ and $<-2.5$ were 1.4 to $1.7,2.1$ to 2.6 and 2.3 to 3.9 times more prevalent among hospitalized versus ambulatory patients at the

Table 3 Osteoporosis diagnosis in ambulatory and hospitalized patients

\begin{tabular}{|c|c|c|c|c|c|c|}
\hline & & \multicolumn{2}{|c|}{ Ambulatory patients } & \multicolumn{2}{|c|}{ Hospitalized patients } & \multirow[t]{2}{*}{$p$-value } \\
\hline & & $N$ & Number (\%) & $\bar{N}$ & Number (\%) & \\
\hline \multirow[t]{3}{*}{ Global osteoporosis } & & 4561 & & 1845 & & $<0.0001$ \\
\hline & No & & 3719 (81.5) & & $1258(68.2)$ & \\
\hline & Yes & & $842(\mathbf{1 8 . 5})$ & & $587(31.8)$ & \\
\hline \multirow[t]{3}{*}{ Lumbar spine osteoporosis } & & 4541 & & 1836 & & $<0.0001$ \\
\hline & No & & $4022(88.6)$ & & $1479(80.6)$ & \\
\hline & Yes & & $519(\mathbf{1 1 . 4})$ & & $357(\mathbf{1 9 . 4 )}$ & \\
\hline \multirow[t]{3}{*}{ Femoral neck osteoporosis } & & 4472 & & 1790 & & $<0.0001$ \\
\hline & No & & 4005 (89.6) & & $1406(78.5)$ & \\
\hline & Yes & & $467(10.4)$ & & $384(\mathbf{2 1 . 5})$ & \\
\hline \multirow[t]{3}{*}{ Total hip osteoporosis } & & 4484 & & 1794 & & $<0.0001$ \\
\hline & No & & 4205 (93.8) & & $1496(83.4)$ & \\
\hline & Yes & & $279(6.2)$ & & $298(\mathbf{1 6 . 6})$ & \\
\hline \multirow[t]{3}{*}{ Osteoporosis at the three sites } & & 4561 & & 1845 & & $<0.0001$ \\
\hline & No & & $4454(97.7)$ & & $1700(92.1)$ & \\
\hline & Yes & & $107(\mathbf{2 . 3})$ & & $145(7.9)$ & \\
\hline
\end{tabular}


Table 4 Demographic and clinical comparison between ambulatory and hospitalized patients

\begin{tabular}{|c|c|c|c|c|c|c|}
\hline & & \multicolumn{2}{|c|}{ Ambulatory patients } & \multicolumn{2}{|c|}{ Hospitalized patients } & \multirow[t]{2}{*}{$p$-value } \\
\hline & & $\bar{N}$ & Mean \pm SD /Number (\%) & $\bar{N}$ & $\overline{\text { Mean } \pm \text { SD } / \text { Number }(\%)}$ & \\
\hline & & & $4561(71.2)$ & & $1845(28.8)$ & \\
\hline Age (years) & & 4561 & $\mathbf{5 9 . 0} \pm 13.2$ & 1845 & $64.2 \pm 16.2$ & $<0.0001$ \\
\hline $\mathrm{BMI}\left(\mathrm{kg} / \mathrm{m}^{2}\right)$ & & 4478 & $25.1 \pm 4.82$ & 1690 & $25.4 \pm 5.79$ & 0.028 \\
\hline \multirow[t]{3}{*}{ Sex } & & 4561 & & 1845 & & $<0.0001$ \\
\hline & $\mathrm{F}$ & & $3571(78.3)$ & & $1196(64.8)$ & \\
\hline & M & & $990(\mathbf{2 1 . 7})$ & & $649(\mathbf{3 5 . 2})$ & \\
\hline \multirow[t]{3}{*}{ GC use } & & 4556 & & 1836 & & 0.078 \\
\hline & No & & $3136(68.8)$ & & $1305(71.1)$ & \\
\hline & Yes & & $1420(31.2)$ & & $531(28.9)$ & \\
\hline \multirow[t]{3}{*}{ PPI intake } & & 2910 & & 1341 & & 0.15 \\
\hline & No & & $2096(72.0)$ & & $937(69.9)$ & \\
\hline & Yes & & $814(28.0)$ & & $404(30.1)$ & \\
\hline \multirow[t]{3}{*}{ Previous fracture } & & 4555 & & 1837 & & $<0.0001$ \\
\hline & No & & $3419(75.1)$ & & $1120(61.0)$ & \\
\hline & Yes & & $1136(\mathbf{2 4 . 9})$ & & 717 (39.0) & \\
\hline
\end{tabular}

lumbar spine, femoral neck and total hip, respectively (Additional file 2: Table S1).

\section{Discussion}

According to our knowledge, this is the first large study evaluating DEXA screening results in a hospitalized subpopulation in comparison to an ambulatory group and demonstrating a higher detection rate of osteoporosis among the in-patient population. Among 6406 patients having a DEXA examination, globally, osteoporosis was detected in $22.3 \%$ of cases. This prevalence is in agreement with a recently published prevalence of $16-38 \%$ based on lumbar spine and of 9-15\% based on total hip BMD [1]. When the cohort was divided into ambulatory ( $n=4561)$ and hospitalized ( $n=1845$ patients), we found striking differences, with hospitalized patients having significantly lower $\mathrm{T}$-scores and having with 2-3 times higher rates of osteoporosis at the lumbar spine, femoral neck and total hip evaluations individually, as well as all three sites. This better detection rate in osteoporosis indicated that an in-hospital screening strategy could be an effective additional tool to improve osteoporosis screening and identification.

As we mentioned, DEXA evaluation was not systematic in any of the group but depended on the physician's judgment. Our study was retrospective and the better detection rate of in-hospital screening could not be due to a selective positive bias. This also revealed the lack of efficient screening in primary care, without any improvement between 2007 and 2012. In another study about osteoporosis screening in inflammatory bowel population, a population at high risk of multifactorial osteoporosis, the screening rate was higher in tertiary referral center compared to secondary center [18]. It's worlwide recognized that osteoporosis secreening is defective. In an ideal world, an optimal screening would be realized in primary care, with a systematic DEXA-scan for woman after menopausis and earlier if there are risk factor for osteoporosis, as proposed by several international guidelines. For man, the consensus is to do not perform DEXA-scan systematicaly, but only if there is a risk factor. For the in-hospital procedure, the same attitude should be proposed if the ambulatory care did not altready perform it. Hospitalization should be the place to think about osteoporosis screening and to perform it if there were no previous screening in ambulatory care.

Hospitalized patients were significantly older with a higher BMI and with more previous fractures than ambulatory patients. GC use and PPI intake were not different in the two sub-populations. Theoretically, older age and more history of major fractures of hospitalized patients could explain the better efficiency. Of interest, only a minor percentage $(5.7 \%)$ of hospitalized patients came from the orthopedic surgery department: the difference between hospitalized and ambulatory patients was consequently not due to hospitalization for an acute fracture. As $78 \%$ of the cohort came from medicine departments, comorbidities or treatment that we could suppose more numerous in this population could also negatively influenced bone mineral density (inflammatory systemic disease with e.g. rheumatoid arthritis [19], polymyalgia rheumatica [20], systemic erythematous lupus [21]; inflammatory bowel disease [22]; mellitus diabetes [23]; renal failure [24]; lung disease [25]...). 
Due to this better positive detection rate, emphasis should be put on the in-hospital osteoporosis screening, because it's the place where much co-morbiditiy accumulate. More systematic screening of the in-hospital population could help to improve osteoporosis detection. In-hospital physicians could also be more concerned or have more time to take care with patients' comorbidities.

In order to neutralize the age parameter, we also decided to compare Z-scores (without age influence): Zscores ranking analysis $(<-1,<-2,<-2.5)$ showed significant difference percentage between ambulatory and hospitalized patients in each of the 3 categories and in each of the three sites studied. Z-scores of the femoral neck and of total hip of hospitalized patients were also significantly lower than those of the ambulatory ones, while differences did not reach statistical signification $(p=0.076)$ at the lumbar spine, although lower. Excess of prevalence in hospitalized patients compared to ambulatory ones increased according to the severity of the category $(<-1,<-2,<-2.5)$ and was maximal in the category Z-score $<2.5$ with nearly four times more in femoral neck and total hip evaluations compared to twice more in lumbar spine evaluation, confirming that differences affected more cortical than trabecular bone. Even if our data were not enough complete in terms of co-morbidities description for each patients, we can hypothesize that trabecular difference would be more influenced by the age difference between the patients and that the cortical difference could be more a reflect of comorbidities linked to inflammatory pre-existing conditions. A limitation of our study was the incomplete characterization in the comorbidities of our patients, that were restricted to the data that we collected that were presented in the results section. Systematic collection of the comorbidities, the drugs and the medical history should be planned to identify which co-morbidities could be associated to the higher rate of osteoporosis in the hospitalized population. In attempt to answer to that question in the future, specific informatic programs that automatically link DEXA data with identification of comorbidities on both sub-populations are currently under development.

Older age, lower BMI and history of fracture (all three in both univariate and multivariate analysis) were classical risk factors present in our study for osteoporosis diagnostic. GC use was another classical osteoporosis risk factor in the univariate analysis at least in one site as well as at the lumbar spine and femoral neck evaluation. In the multivariate analysis however, signification was restricted to the femoral neck evaluation, which is less usual. Explanation could be that GC use is not causal in this situation, but indirectly reflected coexistence of co-morbidities, justification of their use (chronic GC were prescribed in case of inflammatory disease, such as rheumatoid arthritis, polymyalgia rheumatic, vasculitis ... and outside the field of rheumatology with e.g. inflammatory bowel disease, but also after solid or hematopoietic graft or for respiratory disease with asthma or chronic obstructive pulmonary disease). However, we did not have information about dose and duration of GC treatment that could influence the correlation. Male was also associated with OP detection in the univariate analysis at the lumbar spine evaluation. The multivariate analysis confirmed association between male at the lumbar spine but also for osteoporosis diagnostic at least in one of the three sites. Osteoporosis is known to be more common in women and recently it was estimated that women aged 50 years or older have a fourfold higher rate of osteoporosis and a twofold higher rate of osteopenia as compared with men [26]. As a direct consequence, screening was more frequently performed in the female population: e.g. in a primary care center, the screening rate of patients was $60 \%$ for female, but only $18.4 \%$ for men [27] or only $11 \%$ of the eligible men aged 70 years of older in an other study [28]. It must be underlined that there is no real consensus for the screening of men population in the international guidelines. However, male osteoporosis prevalence could be underestimated: a study found a similar prevalence of osteoporosis for men aged 70 years or older and women aged 65 years [27]. After a hip fracture (secondary prevention), osteoporosis screening was also lower in men [29], even if men are known to have a higher rate of complications after an osteoporotic fracture, with a $70 \%$ greater risk of mortality compared with women in a Danish registry [30]. In our cohort, the trend was similar: men only represented $25.6 \%$ of the total population, rising to $35.2 \%$ in the hospitalized population. In univariate analysis, male is associated with osteoporosis at the lumbar spine and this was confirmed in multivariate analysis for the global diagnosis of osteoporosis: men in our population are at greater risk to have osteoporosis than women. An explanation could be that women are more often screened (due to better awareness about osteoporosis risk for women), with less effective screening, whereby only men with established risk factor of osteoporosis were screened. In a study conducted in an inflammatory bowel disease tertiary center, the authors also found that female are more likely to be screened, but male patients were diagnosed more often with osteopenia or osteoporosis than females [31]. Another explanation is that screening is deficient in men leading to underdiagnosis of impaired bone health in men. Recently, an additional paper about patients with inflammatory bowel disease also found that male was a risk factor for osteoporosis [32]. However, in these two papers, osteoporosis was defined as $\mathrm{T}$-score -2.5 at any 
of the site studied and there were no analysis for each site separately, as we have performed.

There exist other limitations in our work: the study was retrospective (and we could only rely on information that were available in the patient's medical record), only compared T-scores between the two sub-populations and did not performed a direct comparison between the bone mass $\left(\mathrm{g} / \mathrm{cm}^{2}\right)$. Nevertheless, this is real life and results were unexpected when we started the study.

\section{Conclusions}

In-hospital osteoporosis screening identified more osteoporosis than in ambulatory practice. Promoting this inhospital detection could be an additional tool to improve osteoporosis managing. This difference is not only due to an older age of the population and suggests the existence of more numerous comorbidities deleterious for bone health in hospitalized patients. In addition to usual risk factors (age, lower BMI, previous fracture), we identify male as associated to osteoporosis detection in our cohort. This underlines the necessity not only to screen women, but also to think about men.

\section{Supplementary information}

Supplementary information accompanies this paper at https://doi.org/10 1186/s12891-020-3116-9.

Additional file 1: Figure S1. Percentage of osteoporotic patients by department in the hospitalized sub-population. The horizontal line represented the mean percentage in the hospitalized sub-population. The percentage in the $X$ axe legend after the name of the department represent the \% of DEXA performed by the department regards to the total number of DEXA performed in the hospitalized sub-population.

Additional file 2: Table S1. Z-score ranking comparison between ambulatory and hospitalized patients

\section{Abbreviations}

BMD: Bone mineral density; BMI: Body mass index; DEXA: Dual energy x-ray absorptiometry; FRAX: Fracture risk assessment tool; GC: Glucocorticoid; OR: Odds ratio; PPI: Proton pump inhibitor; SD: Standard deviation

\section{Acknowledgements}

The authors would like to thank Dr. Adrian Daly for usefull discussion and comments.

\begin{abstract}
Authors' contributions
OM: experimental design, data collection, data analysis, manuscript writing and corrections. MD: data collection, data analysis, manuscript writing and corrections. ML: data collection, data analysis, manuscript corrections. LL: data collection, data analysis, manuscript corrections. LS: data analysis, manuscript writing and corrections. MM: experimental design, data analysis, manuscript writing and corrections. All co-authors take full responsibility for the integrity of the study and all parts of the final manuscript.
\end{abstract}

\section{Funding}

No specific funding was obtained for this study.

\section{Availability of data and materials}

All the data are available form the corresponding author on reasonable request.

\section{Ethics approval}

Approval was obtained from the local ethical committee of the University Hospital of Liège (07/05/2019, reference 2019/152). All procedures performed in studies involving human participants were in accordance with the ethical standards of the institutional research committee and with the 1964 Helsinki declaration and its later amendments or comparable ethical standards.

\section{Consent for publication}

Not applicable.

\section{Competing interests}

Olivier Malaise reports speaker contributions for Amgen and Menarini. Marie Detroz, Mathieu Leroy, Lorenzo Leonori, Laurence Seidel and Michel Malaise declare that they have no conflict of interest.

\section{Author details}

${ }^{1}$ Departments of Rheumatology, University Hospital of Liège, Liège University, Liège, Belgium. ${ }^{2}$ Departments of Biostatistics, University Hospital of Liège, Liège University, Liège, Belgium.

\section{Received: 28 October 2019 Accepted: 4 February 2020}

\section{References}

1. Wade SW, et al. Estimating prevalence of osteoporosis: examples from industrialized countries. Arch Osteoporos. 2014:9:182.

2. Looker AC, et al. Trends in osteoporosis and low bone mass in older US adults, 2005-2006 through 2013-2014. Osteoporos Int. 2017;28(6):1979-88,

3. Marshall $\mathrm{D}$, Johnell $\mathrm{O}$, Wedel $\mathrm{H}$. Meta-analysis of how well measures of bone mineral density predict occurrence of osteoporotic fractures. BMJ. 1996:312(7041):1254-9.

4. Cranney $A$, et al. Low bone mineral density and fracture burden in postmenopausal women. CMAJ. 2007:177(6):575-80.

5. Gillespie CW, Morin PE. Trends and disparities in osteoporosis screening among women in the United States, 2008-2014. Am J Med. 2017;130(3): 306-16

6. Haentjens $P$, et al. Meta-analysis: excess mortality after hip fracture among older women and men. Ann Intern Med. 2010;152(6):380-90.

7. Black DM, et al. Fracture risk reduction with alendronate in women with osteoporosis: the fracture intervention trial. FIT Research Group. J Clin Endocrinol Metab. 2000;85(11):4118-24.

8. Cummings SR, et al. Denosumab for prevention of fractures in postmenopausal women with osteoporosis. N Engl J Med. 2009;361(8): 756-65

9. Kanis JA, et al. FRAX and the assessment of fracture probability in men and women from the UK. Osteoporos Int. 2008;19(4):385-97.

10. Briot K, et al. 2018 update of French recommendations on the management of postmenopausal osteoporosis. Joint Bone Spine. 2018;85(5):519-30.

11. Lafata JE, et al. Improving osteoporosis screening: results from a randomized cluster trial. J Gen Intern Med. 2007:22(3):346-51.

12. Gillespie CW, Morin PE. Osteoporosis-related health services utilization following first hip fracture among a cohort of privately-insured women in the United States, 2008-2014: an observational study. J Bone Miner Res. 2017;32(5):1052-61.

13. Fernandez-Avila DG, et al. Low rate of densitometric diagnosis and treatment in patients with severe osteoporosis in Colombia. Arch Osteoporos. 2019;14(1):95.

14. Nakayama A, et al. Evidence of effectiveness of a fracture liaison service to reduce the re-fracture rate. Osteoporos Int. 2016:27(3):873-9.

15. Brett AD, Brown JK. Quantitative computed tomography and opportunistic bone density screening by dual use of computed tomography scans. J Orthop Translat. 2015;3(4):178-84

16. Carberry GA, et al. Unreported vertebral body compression fractures at abdominal multidetector CT. Radiology. 2013;268(1):120-6.

17. Bruyere $\mathrm{O}$, et al. Comparison of the proportion of patients potentially treated with an anti-osteoporotic drug using the current criteria of the Belgian national social security and the new suggested FRAX criteria. Rheumatol Int. 2013:33(4):973-8.

18. Schule $S$, et al. Widely differing screening and treatment practice for osteoporosis in patients with inflammatory bowel diseases in the Swiss IBD cohort study. Medicine (Baltimore). 2017;96(22):e6788. 
19. Aurrecoechea $\mathrm{E}$, et al. Gender-associated comorbidities in rheumatoid arthritis and their impact on outcome: data from GENIRA. Rheumatol Int. 2017;37(4):479-85.

20. Albrecht $\mathrm{K}$, et al. Long-term glucocorticoid treatment in patients with polymyalgia rheumatica, giant cell arteritis, or both diseases: results from a national rheumatology database. Rheumatol Int. 2018;38(4):569-77.

21. Sato $\mathrm{H}$, et al. High bisphosphonate treatment rates and the prevalence of atypical femoral fractures in patients with systematic lupus erythematosus: a single-center retrospective study performed in Japan. Rheumatol Int. 2019; 39:1803-10.

22. Szafors $P$, et al. Risk of fracture and low bone mineral density in adults with inflammatory bowel diseases. A systematic literature review with metaanalysis. Osteoporos Int. 2018;29(11):2389-97.

23. Tebe $\mathrm{C}$, et al. The association between type 2 diabetes mellitus, hip fracture, and post-hip fracture mortality: a multi-state cohort analysis. Osteoporos Int 2019;30:2407-15.

24. Malmgren $L$, et al. Reduced kidney function is associated with $B M D$, bone loss and markers of mineral homeostasis in older women: a 10-year longitudinal study. Osteoporos Int. 2017;28(12):3463-73.

25. Chen YW, et al. Prevalence and risk factors for osteoporosis in individuals with COPD: a systematic review and meta-analysis. Chest. 2019:156:1092-110

26. Alswat KA. Gender Disparities in Osteoporosis. J Clin Med Res. 2017;9(5): 382-7.

27. Alswat K, Adler SM. Gender differences in osteoporosis screening: retrospective analysis. Arch Osteoporos. 2012;7:311-3.

28. Lim SY, et al. Screening for osteoporosis in men aged 70 years and older in a primary care setting in the United States. Am J Mens Health. 2013;7(4): $350-4$.

29. Antonelli M, Einstadter D, Magrey M. Screening and treatment of osteoporosis after hip fracture: comparison of sex and race. J Clin Densitom. 2014;17(4):479-83.

30. Kannegaard PN, et al. Excess mortality in men compared with women following a hip fracture. National analysis of comedications, comorbidity and survival. Age Ageing. 2010:39(2):203-9.

31. Walldorf $\mathrm{J}$, et al. Health care for osteoporosis in inflammatory bowel disease: unmet needs in care of male patients? J Crohns Colitis. 2013;7(11):901-7.

32. Hoffmann P, et al. Prevalence, risk factors and course of osteoporosis in patients with crohn's disease at a Tertiary Referral Center. J Clin Med. 2019. 8(12). https://doi.org/10.3390/jcm8122178

\section{Publisher's Note}

Springer Nature remains neutral with regard to jurisdictional claims in published maps and institutional affiliations.

Ready to submit your research? Choose BMC and benefit from:

- fast, convenient online submission

- thorough peer review by experienced researchers in your field

- rapid publication on acceptance

- support for research data, including large and complex data types

- gold Open Access which fosters wider collaboration and increased citations

- maximum visibility for your research: over $100 \mathrm{M}$ website views per year

At $\mathrm{BMC}$, research is always in progress.

Learn more biomedcentral.com/submissions 